\title{
Selective recovery of RNAs from bacterial pathogens after their internalization by human host cells
}

\author{
Simon Raynaud ${ }^{1}$, Hélène LePabic ${ }^{1}$ \& Brice Felden $^{1^{*}}$ \\ ${ }^{1}$ Univ Rennes, Inserm, BRM [Bacterial Regulatory RNAs and Medicine] UMR_S 1230, F-35000 \\ Rennes, France.*For correspondence: Email: bfelden@univ-rennes1.fr
}

Highlights

- Method for bacterial RNA recovery from host cells.

- Easy and inexpensive procedure to selectively recover bacterial RNAs from composite cell mix.

- Key influence on beads size and composition during extraction.

\begin{abstract}
Selective RNA extractions are required when studying bacterial gene expression within complex mixtures of pathogens and human cells, during adhesion, internalization and survival within the host. New technologies should be developed and implemented to enrich the amount of bacterial RNAs since the majority of RNAs are from the eukaryotic host cells, requiring high read depth coverage to capture the bacterial transcriptomes in dual-RNAseq studies. This will improve our understanding about bacterial adaptation to the host cell defenses, and about how they will adapt to an intracellular life. Here we present an RNA extraction protocol to selectively enrich the lowest bacterial RNA fraction from a mixture of human and bacterial cells, using Zirconium beads, with minimal RNA degradation. Zirconium beads have higher capacity to extract bacterial RNAs than glass beads after pathogen internalization. We optimized the beads size and composition for an optimal bacterial lysis and RNA extraction. The protocol was validated on two human cell lines, differentiated macrophages and osteoblasts, with either Gram-positive (Staphylococcus aureus) or -negative (Salmonella typhimurium) bacteria. Relative to other published protocols, yield of total RNA recovery was significantly improved, while host cell infection was performed with a lower bacterial inoculum. Within the host, bacterial RNA recovery yields were about six-fold lower than an RNA extraction from pure bacteria, but the quality of the RNA recovered was essentially similar. Bacterial RNA recovery was more efficient for $S$. aureus than for S. typhimurium, probably due to their higher protection by the Gram positive cell walls during the early step of eukaryotic cell lysis. These purified bacterial RNAs allow subsequent genes expression studies in the course of host cellbacteria interactions.
\end{abstract}

Key words: Bacterial \& prokaryotic RNAs, host-pathogen interactions, Zirconium beads, bacterial internalization, RNA expression, selective RNA purification. 


\section{Introduction}

Transcriptomic studies of internalized bacteria gene expression improve our understanding of bacterial adaptation to host cells defense, and intracellular life [1], [2]. Recent RNA-based technologies allow to tackle gene reprograming by intracellular bacterial pathogens when internalized by eukaryotic host cells [3]. Those dual RNA-seq technologies assess how bacterial pathogens fine-tune gene expression when intracellular. Also, information about how the infected host cells react during infection can be collected. A major technical challenge, however, is the huge excess of eukaryotic over bacterial RNA [4] that needs to be removed to obtain specific and reliable transcriptomic data on pathogen gene expression, when intracellular. To this endeavor, extraction methods have been developed to purify RNAs from prokaryotes or eukaryote organisms with higher efficiency and quality [5], [6]. For bacterial RNA extraction from a homogenous population of bacteria, collecting intact, non-degraded RNAs is relatively easy and include mechanical or enzymatic lysis in denaturing conditions, for cell opening [7], [8], followed by phenol extraction of nucleic acids [9] and DNase treatment.

When performed from complex mixtures of organisms, RNA extractions are complicated. However and surprisingly, very few methods are available to extract bacterial RNAs inside eukaryotic cells during infection [10], [11], [12]. Zirconia beads were previously proposed to purify total RNAs for sRNA discovery in bacteria [13]. These strategies provide low amounts of purified RNAs, insufficient for systematic analyses by qPCR or by Northern of bacterial gene expression inside host cells. These methods involve host cells lysis (phenol/ethanol mix; RLT buffer of Qiagen kit, or ice cold acetone: ethanol mix) to leave bacterial cells intact and subsequent total RNA purification. Also, most methods have an enrichment step of the bacterial RNAs with dedicated commercial kit, which increase the cost of the procedure [11], [12]. For S. aureus RNA extraction, most protocols use manufacturer kits [10] [11]. The protocol from [14] lyses bacteria in the bacteria/cellular debris pellets with Trizol and zirconia-silica beads (100 $\mathrm{MM}$ diameter) into a high-speed homogenizer, to isolate total RNA. For S. typhimurium, the first described methods used a mix of $0.1 \%$ SDS, $1 \%$ acidic phenol and $19 \%$ ethanol (in water) to lyse infected epithelial cells [15], [16]. Then, S. typhimurium RNA was prepared using a total RNA purification kit (Promega SV total RNA purification kit). A second method used Trizol to extract RNAs, after host cells lysis with PBS- 0.1\% Triton X-100 [17].

We developed and report here a novel extraction protocol to selectively enrich the minor RNA fraction from internalized bacteria while removing the majority of the eukaryotic RNA excess, using Zirconium beads. We provide evidences that beads sizes and their compositions during extraction have to be optimized to improve bacterial cell lysis for selective bacterial RNA extraction. Bacterial RNA extractions were performed on two human cell lines upon $S$. aureus internalization, THP1-differenciated macrophages and Saos-2 osteoblasts. We also tested the new method for THP1-differenciated macrophages infected with S. typhimurium. We anticipate our bacterial RNA purification protocol to be 
applicable for various bacterial-host cell gene expressions, for subsequent transcriptomic studies.

\section{Methods}

\subsection{Bacteria, human cells and cultures.}

RNA extraction of Staphylococcus aureus (Newman) [18] and Salmonella typhimurium strains were selected as Gram-positive and -negative representatives. The two strains were grown at $37^{\circ} \mathrm{C}$ in Brain Heart Infusion broth (BHI, Oxoid, Dardilly, France) for S. aureus, and in Lysogeny Broth (LB, Sigma, Saint-Louis, USA) containing $300 \mathrm{mM} \mathrm{NaCl}$ for S. typhimurium.

THP1 human monocytes were from ATCC (Rockville, USA) and maintained at $37^{\circ} \mathrm{C}$ with $5 \% \mathrm{CO}_{2}$ in RPMI1640 (Thermo Fisher Scientific, Waltham, USA) containing 10\% FCS (GE Healthcare Hyclone, Chicago, USA). The THP1 cells were treated with 20ng/ml phorbol 12-myristate 13-acetate (PMA, Sigma, St Louis, USA) for 3 days for differentiation into macrophages. The THP1 monocytes were differentiated into adherent macrophages, as evidenced by light microscopy. Saos-2 human osteoblasts (ATCC, Rockville, USA), are maintained at $37^{\circ} \mathrm{C}$ with $5 \% \mathrm{CO}_{2}$ in McCoy medium (Thermo Fisher Scientific's, Waltham, USA) containing 15\% FCS (GE Healthcare Hyclone, Chicago, USA).

\subsection{Bacterial internalization and human cell lysis.}

Human THP1-differenciated macrophages and Saos-2 osteoblasts were infected at a multiplicity of infection (MOI) of 1:10 and 1:30, respectively, with either S. aureus or S. typhimurium. Experiments were conducted in 6-wells plates with $5.10^{5}$ THP1-differenciated macrophages, or $4.10^{5}$ Saos-2 osteoblasts by well. Internalized assays of S. aureus were carried out for $2 \mathrm{~h}$ at $37^{\circ} \mathrm{C}$, with $5 \% \mathrm{CO}_{2}$ in RPMI 1640 containing $10 \%$ of human SAB for THP1-differenciated macrophages and in McCoy medium with $15 \%$ of human SAB for Saos-2 osteoblasts. Internalization was stopped on ice and cells were washed three times with ice-cold PBS. All the non-internalized bacteria were removed by culturing the human cells overnight in a medium supplemented with $50 \mu \mathrm{g} / \mathrm{ml}$ gentamycin (Sigma, St Louis, USA).

Internalized assays of $S$. typhimurium were carried out for $2 \mathrm{~h}$ at $37^{\circ} \mathrm{C}$, with $5 \%$ $\mathrm{CO}_{2}$ in RPMI 1640 containing 10\% of human SAB for THP1-differenciated macrophages. After washing the cells three times with PBS, all the non-internalized bacteria were removed by culturing the human cells for $90 \mathrm{~min}$ in a medium supplemented with $100 \mu \mathrm{g} / \mathrm{ml}$ gentamycin (Sigma, St Louis, USA). Subsequently, for both internalized assays, the medium was removed, cells were washed three times 
with PBS and incubated with $500 \mu \mathrm{L}$ of PBS/SDS $1 \%$ for $15 \mathrm{~min}$ at room temperature for cell lysis. Three or six wells were pooled to increase the amount of RNA extracted for each condition, and then centrifuged. After centrifugation, supernatant containing intracellular material of human cells was discarded.

\subsection{RNA extraction}

A method is described for selective bacterial RNA extraction by phenol/chloroform after human cells lysis and mechanical disruption of the bacterial envelopes with beads. The mechanical lysis probably improves bacterial release from the eukaryotic cells.

\subsubsection{The beads}

Four different types of beads were tested for their abilities to extract RNAs from internalized bacteria. Glass beads (Sigma Chemical Co., St. Louis, USA), used as references, were compared to four new types of beads (Dutscher, Brumath, France). Table 1 shows their compositions, diameters and densities. The variation of beads composition and density should influence RNA extraction. Also, the variation of beads size should impact bacterial crushing, especially those trapped into the eukaryotic cell debris. Each bead set was washed twice in purified water and then twice in an RNA extraction buffer (SDS 0.5\%; 20 mM sodium acetate; 1 mM EDTA pH 5.5), before use.

\begin{tabular}{|c|c|c|c|}
\cline { 2 - 4 } \multicolumn{1}{c|}{ Beads } & Compositions & Diameters $(\mu \mathrm{M})$ & Densities $\left(\mathrm{g} / \mathrm{cm}^{3}\right)$ \\
\hline 1 & Zirconium & 100 & 6.5 \\
\hline 2 & Zirconium & 400 & 6.5 \\
\hline 3 & Steel & 200 & 7.8 \\
\hline 4 & Zirconium oxyde & 500 & 5.9 \\
\hline 5 & Glass & 100 & 2.5 \\
\hline
\end{tabular}

Table 1: Tested beads, physical and chemical properties

\subsubsection{RNA extraction from host cell-internalized bacteria.}

(1) Prepare the Fast Prep tubes (cryotubes): add $250 \mu \mathrm{L}$ beating beads (one of the five from Table 1), $250 \mu \mathrm{L}$ phenol $(\mathrm{pH} 4)$ and $250 \mu \mathrm{L}$ of a $\mathrm{CHCl}_{3} /$ Isoamyl alcohol $\operatorname{mix}(24: 1)$.

(2) Mix the bacteria/THP1-differenciated macrophages or the bacteria/Saos-2 osteoblasts lysates from the internalization assays in $500 \mu \mathrm{L}$ of RNA extraction 
buffer. Transfer this solution into a cryotube containing the beating beads and lyse into a Fast Prep, 30sec, power 6.5. As negative control, the same experiment was done with a bacteria pellet only, without human cells.

(3) Centrifuge cells at $12000 \mathrm{rpm}$ for $5 \mathrm{~min}$ at $4^{\circ} \mathrm{C}$, and keep the supernatant.

(4) Extraction 'volume-to-volume' with a Phenol/CHCl3/Isoamyl alcohol mix (25:24:1).

(5) Centrifuge the mix at $12000 \mathrm{rpm}$ for 5 min at $4^{\circ} \mathrm{C}$, and keep the upper phase.

(6) Extract 'volume-to-volume' with a $\mathrm{CHCl} 3 /$ Isoamyl alcohol mix (24:1).

(7) Centrifuge the mix at $12000 \mathrm{rpm}$ for 5 min at $4^{\circ} \mathrm{C}$, and keep the upper phase.

(8) Precipitate the RNAs with $0.7 \mathrm{vol}$ of isopropanol and $0.1 \mathrm{vol}$ of sodium acetate $3 \mathrm{M}(\mathrm{pH} 5.2)$, for $2 \mathrm{~h}$ at $-80^{\circ} \mathrm{C}$.

(9) Centrifuge the mix at $12000 \mathrm{rpm}$ for $30 \mathrm{~min}$ at $4^{\circ} \mathrm{C}$, discard the supernatant.

(10) Wash the pellet with $500 \mu \mathrm{L}$ of $70 \%$ ethanol.

(11) Centrifuge the RNAs at $12000 \mathrm{rpm}$ for $5 \mathrm{~min}$ at $4^{\circ} \mathrm{C}$, discard the pellet.

(12) Dry the pellet for 5 min into a Speed Vacuum and dissolve the pellet in RNaseDNase free water.

\subsubsection{Human cell RNA extraction.}

RNAs from the THP1-differenciated macrophages were extracted by using an RNeasy ${ }^{\circledR}$ Mini Kit (Qiagen, Venlo, Netherland) in accordance with the protocol from the supplier. No gentamycin treatment was applied to this sample, which only serves as an internal control (eukaryotic rRNAs).

\subsection{Quality-control assessment of the extracted RNAs.}

\subsubsection{Gel electrophoresis}

(1) Each sample of RNAs extracted from the internalized bacterial, prokaryotic and eukaryotic RNA control was diluted into $10 \mu \mathrm{L}$ final volume, and $2 \mu \mathrm{L}$ of $6 \mathrm{X}$ gel loading dye were added (NEB, Evry, France).

(2) Load and migrate the samples for $1 \mathrm{~h}$ at $90 \mathrm{~V}$ on $1 \%$ agarose gel, supplemented with ethidium bromide. 
(3) Visualize the eukaryotic $28 \mathrm{~S}$ and $18 \mathrm{~S}$ rRNAs and the prokaryotic $23 \mathrm{~S}$ and $16 \mathrm{~S}$ rRNAs by UV transilluminator (Figs 1 and 2 ).

\subsubsection{RNA6000 Nano assays}

RNA Nano Chips (Agilent, Santa Clara, USA) were used to quantify RNAs and access RNA Integrity Numbers (RIN, Table 3). The RNA Nano Chips were prepared according to the protocol supplied with the RNA 6000 Nano assay kit (Agilent).

(1) Decontaminate the electrodes with an electrode cleaner chip filled with an RNaseZAP ${ }^{T M}$ RNase decontamination solution (Ambion, Inc. cat. No. 9780) for 1 min and once with an electrode cleaner chip filled with RNase-free water for $10 \mathrm{sec}$.

(2) The chip was filled with the gel-Dye Mix (Agilent).

(3) $5 \mu \mathrm{L}$ of RNA markers mixture was then applied to each well, together with $1 \mu \mathrm{L}$ of a heat denatured $\left(2 \mathrm{~min}\right.$ at $70^{\circ} \mathrm{C}$ ) sample or an RNA ladder (Agilent).

(4) The chip was vortexed and run on the Agilent 2100 Bioanalyzer.

\subsection{Assessing the amount and ratio of prokaryotic versus eukaryotic RNAs.}

The amount and proportion of eukaryotic versus prokaryotic RNAs in either the THP1-differenciated macrophages or Saos-2 osteoblasts host cells with internalized bacteria was independently monitored by (i) RT-qPCR, (ii) gel electrophoresis and (iii) bioanalyzer.

(1) Remove putative DNA remnants from the extracted RNAs using amplificationgrade DNase I (Life Technologies, Carlsbad, USA).

(2) Synthesize cDNAs with a High-Capacity cDNA Reverse Transcriptase Kit (Life Technologies, Carlsbad, USA).

(3) Perform qPCR using a Real Master Mix SYBR Kit (5' PRIME, Life Technologies, Carlsbad, USA) on a StepOnePlus Real-Time PCR system (GE Healthcare, Saint Aubin, France). The DNA Primers are listed on Table 2. 


\begin{tabular}{|c|c|c|}
\hline Detected RNAs & Primers & $\left(5^{\prime}-3^{\prime}\right)$ sequences \\
\hline \multirow{2}{*}{$\begin{array}{l}\text { invA mRNA ( } S . \\
\text { typhimurium) }\end{array}$} & invA-F & CAACGTTTCCTGCGGTACTGT \\
\hline & invA-R & CCCGAACGTGGCGATAATT \\
\hline \multirow{2}{*}{ tmRNA (S. aureus) } & tmRNA-F & CACTCTGCATCGCCTAACAG \\
\hline & tmRNA-R & GATTTGAACCCGCGTCCAG \\
\hline \multirow{2}{*}{$\begin{array}{c}\text { 16S rRNA (S. aureus \& } \\
\text { S. typhimurium) }\end{array}$} & $16 S-F$ & GTTGCGGGACTTAACCCAAC \\
\hline & $16 S-R$ & GCAACGCGAAGAACCTTACC \\
\hline \multirow{2}{*}{ 18S rRNA (human) } & $18 S-F$ & CGCCGCTAGAGGTGAAATTC \\
\hline & $18 S-R$ & TTGGCAAATGCTTTCGCT \\
\hline \multirow{2}{*}{ gapdH mRNA (human) } & Gapdh-F & TGCACCACCAACTGCTAAGC \\
\hline & Gapdh-R & GGCATGGACTGTGGTCATGAG \\
\hline
\end{tabular}

Table 2: Sequences of the DNA primers used to detect bacterial and host cells RNAs by qPCR.

For the gene expression studies targeting invA mRNA, tmRNA, 18S rRNA and gapdH mRNA, a Ct 'cut-off value' of 35 was chosen because obtaining a 3-cycle difference in target $\mathrm{Ct}$, in comparison to a negative control $\mathrm{Ct}$, except for the 16S rRNA expression where the $\mathrm{Ct}$ 'cut-off value' of 28 was selected. Before scoring any reaction, optimized qPCR conditions and melting curves were conducted by verifying that the $\mathrm{Ct}$ values and amplification curves were both acceptable. As the assay's thermal cycling protocol involved 40 cycles, Ct 35 was the latest Ct value where the above requirements were acceptable [19].

\section{Results and discussion.}

After human cell internalization, bacterial RNA extraction allows studying bacterial gene expression within various host cells. Here we propose an easy and inexpensive procedure to increase bacterial RNA recovery while removing most of the eukaryotic RNA excess after host cell uptake. The technology was tested on two human cell types infected by either Grampositive of -negative bacteria, used as model organisms. Various types of beating beads were tested to improve the purification of prokaryotic RNAs, especially those protected and trapped within the eukaryotic cells debris. 


\subsection{Beads testing and selection.}

In this proposed method, S. aureus strain Newman was internalized by two different human cell lines, THP1-differienciated macrophages and Saos-2 osteoblasts. These uptakes were followed by cells lysis and total RNA extraction by using five different types of beads (Table 1). As shown in Figure 1, two samples were compared for each type of beads. Extraction controls were achieved with non-internalized bacteria $\left(3.10^{7}\right)$ compared to macrophages-internalized bacteria $\left(3.10^{6}\right.$ human cells with $3.10^{7}$ bacteria; $\left.\mathrm{MOI} 1: 10\right)$. In Figure 1, we see two bands correspond to the $23 \mathrm{~S}$ and $16 \mathrm{~S}$ rRNAs extracted from the prokaryotic controls, as well as two bands corresponding to the $28 \mathrm{~S}$ and $18 \mathrm{~S}$ rRNAs extracted from the eukaryotic control. For RNAs extracted from internalized bacteria with beads 1 or 4 , we detect the intact bacterial rRNAs, with no or low amounts of human rRNAs. However, beads 2, 3 and 5 are inappropriate for bacterial RNA recovery after host cell internalization (Figure 1). Beads 1 were selected based on the amount of RNAs collected estimated by (i) Nanodrop dosage, (ii) visual integrity on agarose gel and also according to the beads diameter that is identical to that of glass beads, adapted for bacterial lysis (Table 3 and Figure 1).

\begin{tabular}{|c|c|c|c|}
\cline { 2 - 4 } \multicolumn{1}{c|}{} & \multicolumn{1}{c}{ Beads } & $\begin{array}{c}\text { Yields of prokaryotic } \\
\text { control RNA recovery }\end{array}$ & $\begin{array}{c}\text { Yields of total RNA } \\
\text { recovery }\end{array}$ \\
\hline Beads 1 & $100 \mu \mathrm{m}$ Zirconium beads & $35-40 \mu \mathrm{g}$ & $20-30 \mu \mathrm{g}$ \\
\hline Beads 2 & $400 \mu \mathrm{m}$ Zirconium beads & $10-12 \mu \mathrm{g}$ & $2-5 \mu \mathrm{g}$ \\
\hline Beads 3 & $200 \mu \mathrm{m}$ Steel beads & $24-40 \mu \mathrm{g}$ & $10-15 \mu \mathrm{g}$ \\
\hline Beads 4 & $500 \mu \mathrm{m}$ Zirconium Oxide beads & $25-30 \mu \mathrm{g}$ & $20-30 \mu \mathrm{g}$ \\
\hline Beads 5 & $100 \mu \mathrm{m}$ Glass beads & $20-40 \mu \mathrm{g}$ & $0.8-1.4 \mu \mathrm{g}$ \\
\hline
\end{tabular}

Table 3. Total RNA recovery from bacteria internalized into human cells and the respective controls testing various beads for cell crushing. The experiment is the result of pooling 6 wells which correspond to $3.10^{6}$ human cells and $3.10^{7}$ bacteria. The RNA dosage were done by Nanodrop. 


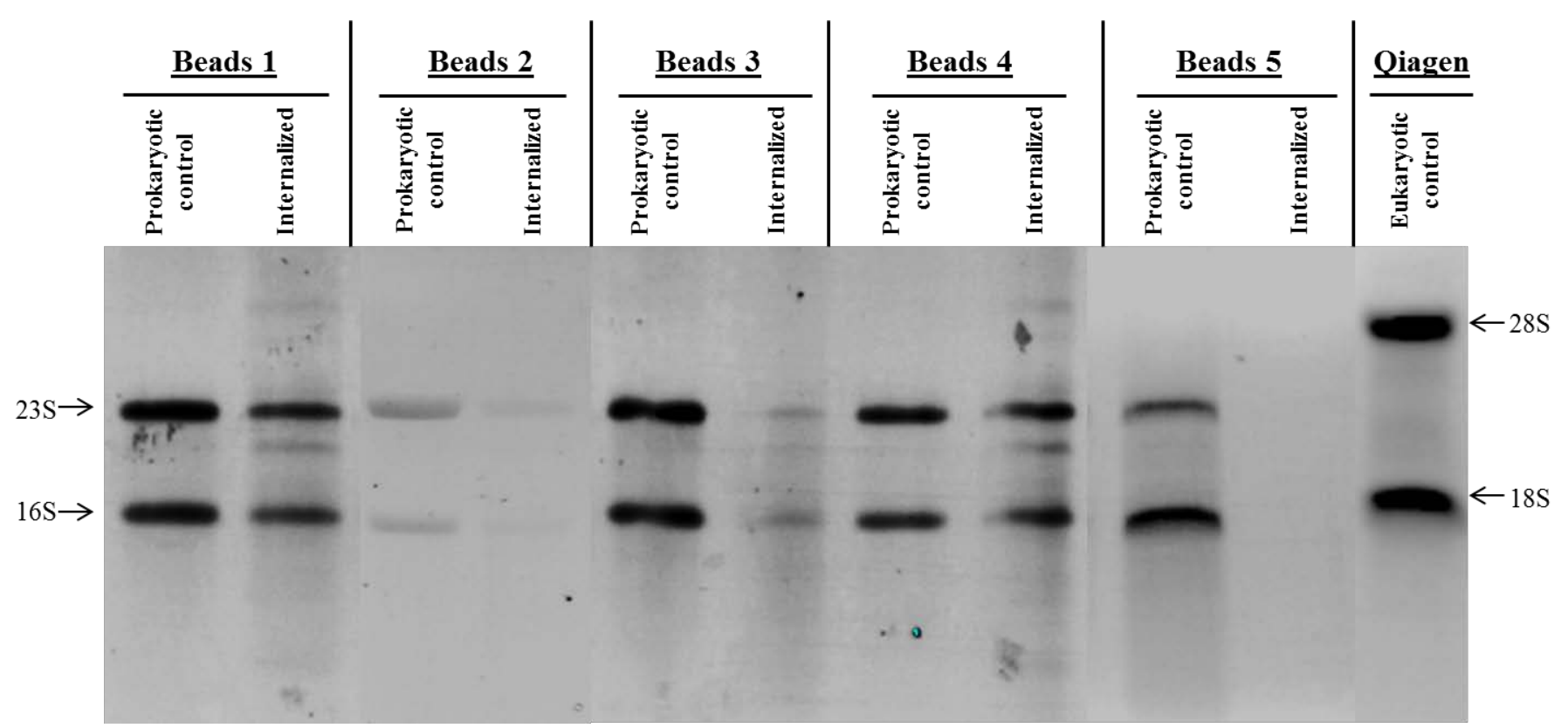

Figure 1: Monitoring the extracted RNAs after bacterial ( $S$. aureus Newman, 500ng total RNA) uptake by human macrophages. Agarose gel stained with ethidium bromide. $23 \mathrm{~S}$ and 165 rRNAs are the prokaryotic rRNAs whereas the eukaryotic rRNAs are undetectable. Prokaryotic controls represent S. aureus Newman cells only. The eukaryotic control represents THP1-differenciated macrophages cells only.

\subsection{Comparison between Glass and Zirconium beads.}

To investigate the efficiency of the zirconium beads compared to the glass beads, we performed the RNA extraction in parallel to compare these two types of beads. The extracted RNAs were analyzed by an electrophoresis on agarose gel (Figure 2). For the 'non-infected macrophage' control, an extraction was performed with RNeasy ${ }^{\circledR}$ Mini Kit (Quiagen, Venlo, Netherland).

First, for the S. aureus prokaryotic control (Newman strain), two bands with no degradation correspond to the $23 \mathrm{~S}$ and the $16 \mathrm{~S}$ rRNAs, and attest RNA integrity (Figure $2 \mathrm{~A})$ with both the glass and zirconium beads. Second, for the non-infected conditions (THP1-differenciated macrophages), two bands are detected corresponding to the 28S and the $18 \mathrm{~S}$ rRNAs, attesting the efficiency of intact eukaryotic RNAs recovery with the Qiagen kit (Figure 2A). This sample serves as positive control. For the internalized bacteria, we detected differences between the THP1 and Saos-2 host cells. For the macrophages-internalized bacteria, we purified intact bacterial RNAs with the Zirconium beads, but not with the glass beads (Figure 2A). For the Saos-2-internalized bacteria, however, we collected both the bacterial and eukaryotic rRNAs with the zirconium beads (Figure 2A). Our hypothesis is that during THP1 cells lysis, most of the eukaryotic RNAs are released and discarded since they locate in the supernatants after centrifugation, whereas the prokaryotic RNAs is probably sheltered inside bacteria (pellet). For the Saos- 
2 cells, their lysis may be less effective, with a lower release and elimination of their (eukaryotic) RNAs.

For the RNAs extracted from Gram-negative S. typhimurium (control), we detected three bands corresponding to the $23 \mathrm{~S}$ rRNA, which is cleaved in two smaller RNA fragments of 1.1 and $1.7 \mathrm{~kb}$ [20], together with full-length $16 \mathrm{~S}$ rRNAs for both the glass and zirconium beads, validating the control (Figure $2 \mathrm{~B}$ ). We used the same sample from Figure $2 \mathrm{~A}$ for a eukaryotic control. For the internalized bacteria, we visualize a lower quantity of RNAs than for $S$. aureus, as well as more contaminating eukaryotic RNAs (Table 4 and 5). There is a higher yield of RNA recovery with the zirconium beads than for glass beads. The extraction is less effective for the Gram-negative bacteria than for the Gram-positive.

In conclusion, zirconium beads have higher capacity to extract bacterial RNAs than glass beads after pathogen internalization. This works better for Gram-positive (S. aureus) in comparison to Gram-negative bacteria ( $S$. typhimurium). We assume that the improvement of extraction was due to the elevated density of zirconium ( $d=6.5)$ in comparison to glass $(d=2.5)$. This characteristic may allow efficient bacteria crushing after their internalization by both THP1 and Saos-2 cells.

\begin{tabular}{|c|c|c|c|c|}
\hline & & $\begin{array}{l}\text { RNA concentration } \\
(\mathrm{ng} / \mu \mathrm{L})\end{array}$ & $\begin{array}{c}\text { Total RNA } \\
\text { recovered } \\
(\mu \mathrm{g})\end{array}$ & Loaded RNA (ng) \\
\hline \multirow{2}{*}{$\begin{array}{c}\text { Prokaryotic control } \\
\text { (S. aureus) }\end{array}$} & Glass & 3250 & 97 & 1620 \\
\hline & Zirconium & 6900 & 207 & 3450 \\
\hline \multirow{2}{*}{$\begin{array}{l}\text { Prokaryotic control } \\
\text { (S. typhimurium) }\end{array}$} & Glass & 1350 & 21.6 & 400 \\
\hline & Zirconium & 1650 & 26.4 & 400 \\
\hline $\begin{array}{c}\text { Eukaryotic control (THP1 } \\
\text { macrophages) }\end{array}$ & Qiagen & 716 & 21.5 & 400 \\
\hline \multirow{2}{*}{$\begin{array}{c}\text { THP1-internalized } \\
\text { (S. aureus) }\end{array}$} & Glass & 2.9 & 0.06 & 29 \\
\hline & Zirconium & 1070 & 17 & 300 \\
\hline \multirow{2}{*}{$\begin{array}{l}\text { Saos-2-internalized } \\
\text { (S. aureus) }\end{array}$} & Glass & 26 & 0.5 & 300 \\
\hline & Zirconium & 62 & 1.25 & 300 \\
\hline \multirow{2}{*}{$\begin{array}{l}\text { THP1-internalized } \\
\text { (S. typhimurium) }\end{array}$} & Glass & 12 & 190 & 120 \\
\hline & Zirconium & 128 & 1540 & 300 \\
\hline
\end{tabular}

Table 4. Comparing total RNA recovery (Nanodrop dosage) between glass and Zirconium

beads. The amounts of loaded RNA correspond to the data presented in Figure 2. 


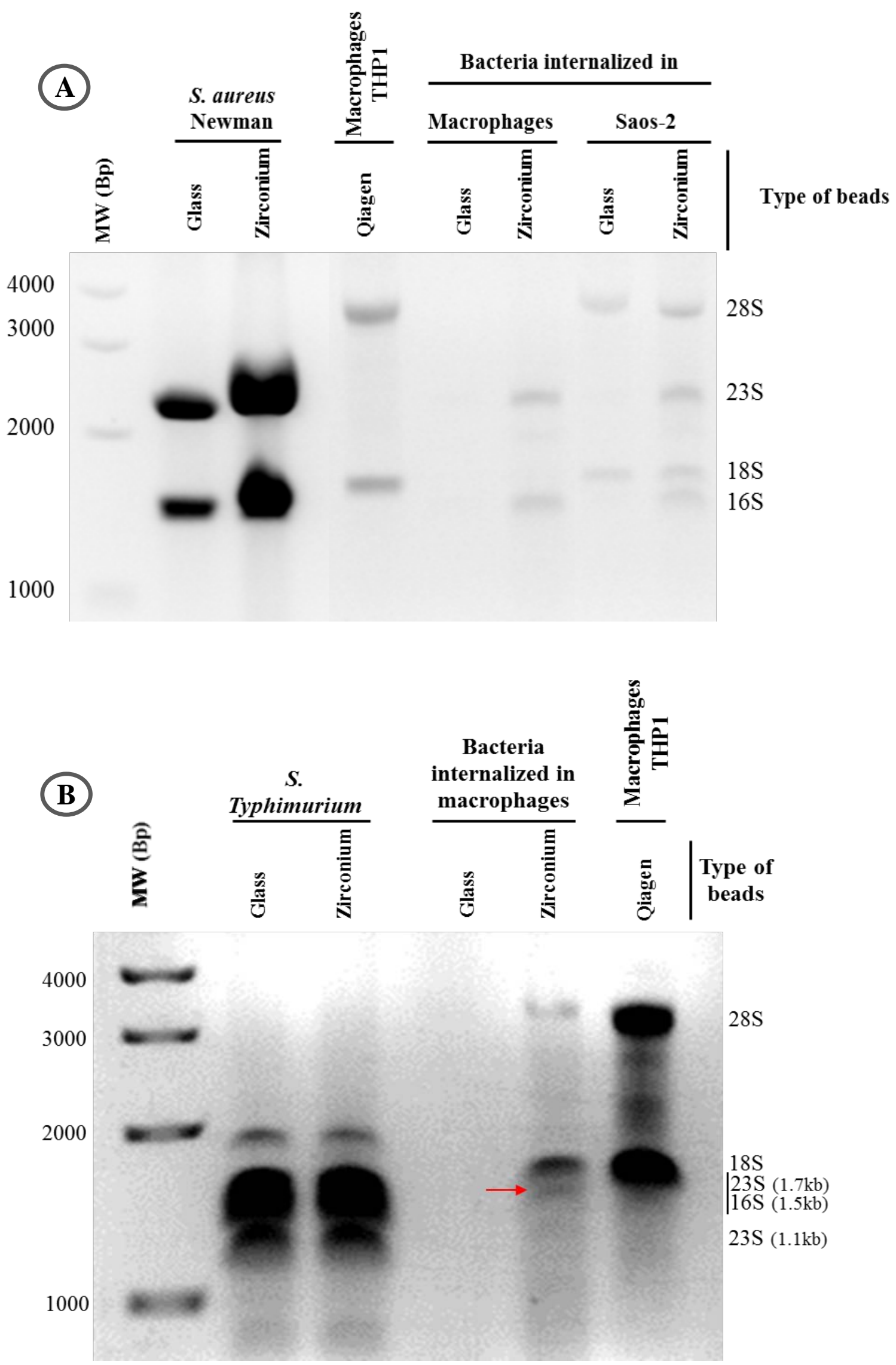


Figure 2: Monitoring the extracted RNAs from internalized bacteria by agarose gel electrophoresis stained with ethidium bromide. 23S and 16S rRNAs reveal the presence of prokaryotic RNAs. $28 \mathrm{~S}$ and $18 \mathrm{~S}$ rRNAs are the contaminating eukaryotic RNAs. The eukaryotic control represents RNAs extracted from THP1-differenciated macrophages cells. A, prokaryotic controls represent RNAs extracted from S. aureus Newman cells. B, prokaryotic controls represent RNAs extracted from S. typhimurium cells. The arrow points to the bacterial rRNA. The amounts of RNA loaded are indicated onto Table 4.

\subsection{Quality control of the extracted RNAs.}

Next, we performed a RNA chip to monitor the quality of the extracted RNAs after THP1 uptake (Table 5). The chip allows quantifying the extracted RNAs to assess their integrity. For each condition (prokaryote control, Saos-2 internalized bacteria and THP1 internalized bacteria), higher amount of RNAs was collected with the zirconium beads compared to the glass beads (Table 5). Also, the RNAs extracted from THP1 internalized $S$ aureus with zirconium beads show a RNA Integrity Number (RIN) of 8.7, compared with the prokaryotic controls (RIN of 9.6). That RIN validated the integrity of the extracted RNAs, except for a slight degradation of 235 rRNA for the internalized S. aureus (Supplemental data Figure A.1). For the Saos-2 internalized bacteria, with the RNAs extracted by the Zirconium beads, we obtained a mixture of eukaryotic and prokaryotic RNAs. On the electrophoregram of the extracted RNAs from the Saos-2 internalized bacteria (Supplemental data Figure A.2), 4 peaks correspond to the eukaryotic and prokaryotic rRNAs, and one peak may correspond to a $23 \mathrm{~S}$ rRNA degradation, as for the THP1 internalized bacteria. This electrophoregram attests of the integrity of the extracted RNAs, with each peak associated to an rRNA (Supplemental data Figure A.2). A summary of each extracts dropped on the chip is represented on Figure B.1 (Supplemental data).

We realized a second RNA chip to get information about the quality of our $S$. typhimurium extracts. The RIN of both glass and zirconium beads are 6.4 and 6.2 respectively. These values are lower than for $S$. aureus and can be due to the $23 S$ rRNA cleavage [20] that we discussed earlier. A profile similar to that obtained by [15] was observed, with three bands. One of them corresponding to the 16S rRNA and the two others to $23 S$ rRNA fragments (Supplemental data B.2). For the THP1-internalized S. typhimurium, a mix of eukaryotic and prokaryotic RNAs is detected (RIN of 5.2), which can be consider as acceptable in comparison to prokaryotic control RIN. On the electrophoregram (Supplemental data A.3), we could detect easily the prokaryotic rRNAs and validate their presence in our extracts. 


\begin{tabular}{|c|c|c|c|}
\hline \multicolumn{2}{|l|}{ Extracted RNAs } & Total Amount ( $\mu \mathrm{g})$ & RNA Integrity Number \\
\hline \multirow{2}{*}{$\begin{array}{l}\text { Prokaryotic control } \\
\text { (S. aureus) }\end{array}$} & Glass & 97 & 9.6 \\
\hline & Zirconium & 207 & 9.6 \\
\hline \multirow{2}{*}{$\begin{array}{l}\text { Prokaryotic control } \\
\text { (S. typhimurium) }\end{array}$} & Glass & 21.6 & 6.4 \\
\hline & Zirconium & 26.4 & 6.2 \\
\hline $\begin{array}{l}\text { Eukaryotic control } \\
\text { (THP1 macrophages) }\end{array}$ & Qiagen & 21.5 & 9.5 \\
\hline \multirow{2}{*}{$\begin{array}{l}\text { THP1-internalized } \\
\text { (S. aureus) }\end{array}$} & Glass & 0.12 & N/A \\
\hline & Zirconium & 32.1 & 8.7 \\
\hline \multirow{2}{*}{$\begin{array}{c}\text { Saos-2-internalized } \\
\text { (S. aureus) }\end{array}$} & Glass & 0.5 & N/A \\
\hline & Zirconium & 1.25 & N/A \\
\hline \multirow{2}{*}{$\begin{array}{l}\text { THP1-internalized } \\
\text { (S. typhimurium) }\end{array}$} & Glass & 0.06 & N/A \\
\hline & Zirconium & 0.35 & 5.2 \\
\hline
\end{tabular}

Table 5. Qualitative and quantitative analyses of the extracted RNAs from $S$. aureus Newman or $S$. typhimurium uptake by human cells. The experiment results in pooling 6 wells, which correspond to $3.10^{6}$ human cells and $3.10^{7}$ bacteria. RIN: N/A = Not Attributed, ' 1 ' corresponds to degraded RNAs, and ' 10 ' to intact RNAs. The bacterial inoculum was $50 \mu \mathrm{l}\left(5.10^{6} \mathrm{CFU}\right)$ per well of $2 \mathrm{ml}$, corresponding to a total inoculum of $300 \mu \mathrm{l}$.

\subsection{Assessing eukaryotic and prokaryotic RNA expression from the extracts.}

Gene expression studies were performed on total RNAs extracted with the $100 \mu \mathrm{M}$ diameter Zirconium beads. The expression levels of selected RNA genes were monitored by RT-qPCR in the RNAs extracted from THP1 internalized S. aureus Newman and $S$. typhimurium. The expression of several representative genes was measured: gapdH mRNA and 18S rRNA were recorded from RNAs extracted from THP1-differentiated cells, tmRNA from RNAs extracted from S. aureus Newman, invA from RNAs extracted from $S$. typhimurium, and 16S rRNA (extracted from both S. aureus and S. typhimurium) expression levels.

First, we studied the expression of $16 \mathrm{~S}$ rRNA, a common gene between $S$. aureus and S. typhimurium. We designed a set of primers that could work on both bacteria, amplifying a conserved region of the RNA.

The Ct value of a S. aureus RNA control is lower than that of a S. typhimurium control, when monitoring 16s RNA expression (Fig 3A). This difference between the two strains is also detected when comparing the values obtained when intracellular. The Ct value of 
$16 \mathrm{~S}$ rRNA detection increases for both bacteria when intracellular, as expected. We also monitored RNA expression for a gene specific for each bacterium. For $S$. aureus, we monitored tmRNA expression (Fig. 3B), which was reported as unfluctuating before and after $S$. aureus uptake [21], that is also observed here. For S. typhimurium, genus-specific invA mRNA was selected and was detected when the bacteria are internalized (Figure 3C). These expression data are in agreement with both the agarose gel and the chip experiments. Overall, our RNA extraction method is efficient for Gram-positive bacteria during host cell uptake but, to a lesser extent, for a Gram-negative bacterium. Probably the thicker, solid envelope of $S$. aureus better protects the RNA during the initial lysis steps of the eukaryotic cells than for $S$. typhimurium.
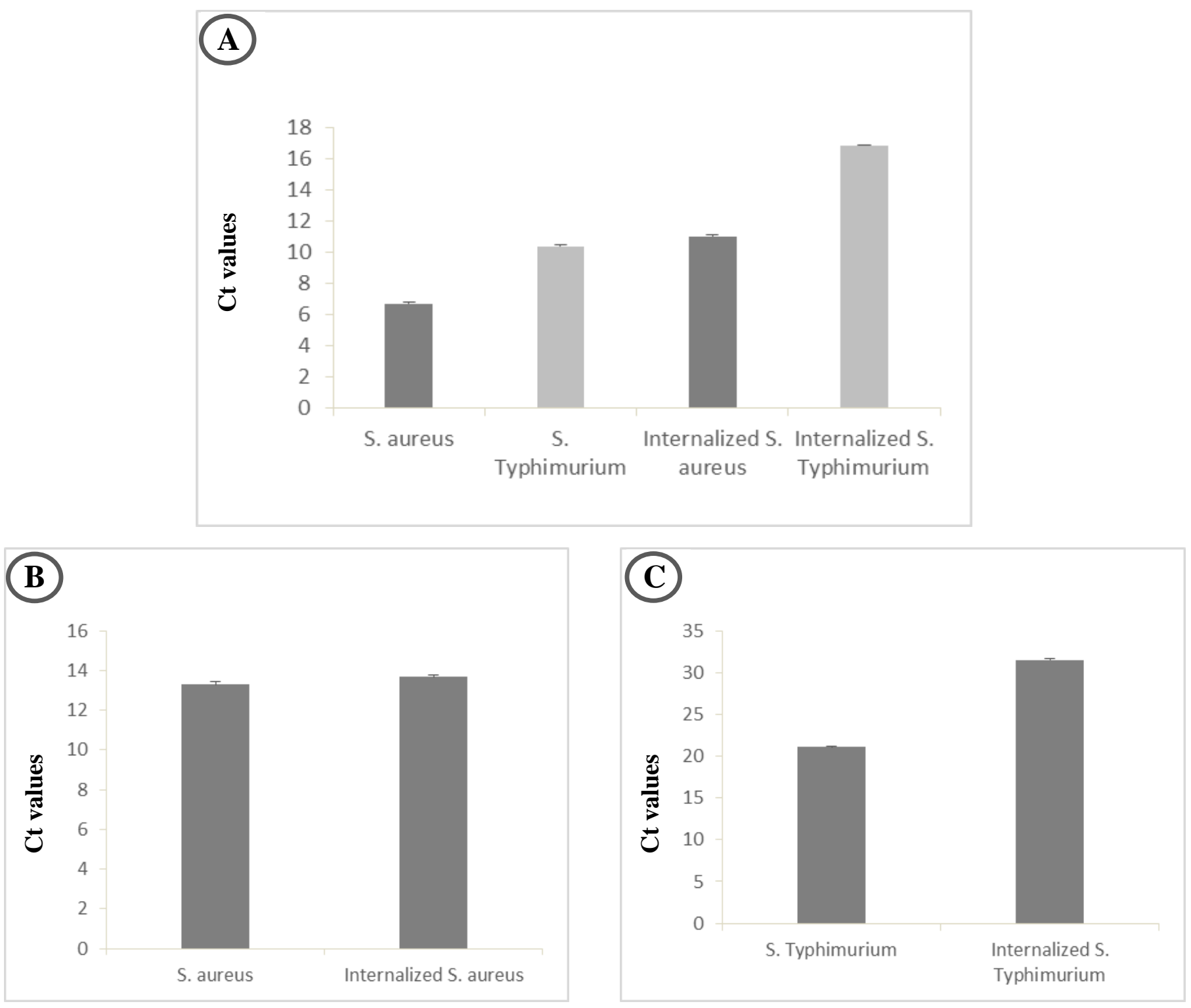

Figure 3: RNA expression levels of selected prokaryotic genes in internalized bacteria versus non-internalized bacteria by RT-qPCR. The primer set to amplify the $16 S$ rRNA are identical for both bacteria (A), tmRNA is specific to $S$. aureus (B) and InvA mRNA is specific to $S$. typhimurium (C). 
Monitoring the RNA expression levels of selected eukaryotic genes inform us about the amount of eukaryotic RNAs still present in the RNA extracts from internalized bacteria, compare with a non-infected 'macrophages only', sample. First, in Figure 4A, we studied $18 \mathrm{~S}$ rRNA expression which is highly expressed (a Ct value of 7.6 in macrophages). In comparison, the $\mathrm{Ct}$ values of internalized S. aureus and S. typhimurium are higher (15.8 and 10.9) respectively. It indicates that the amount of eukaryotic rRNA was reduced in our internalized sample, with a less efficiency for S. typhimurium. For mRNA, we studied expression of gapdH mRNA and obtained the same profile than for the 18S rRNA (Fig 4B).
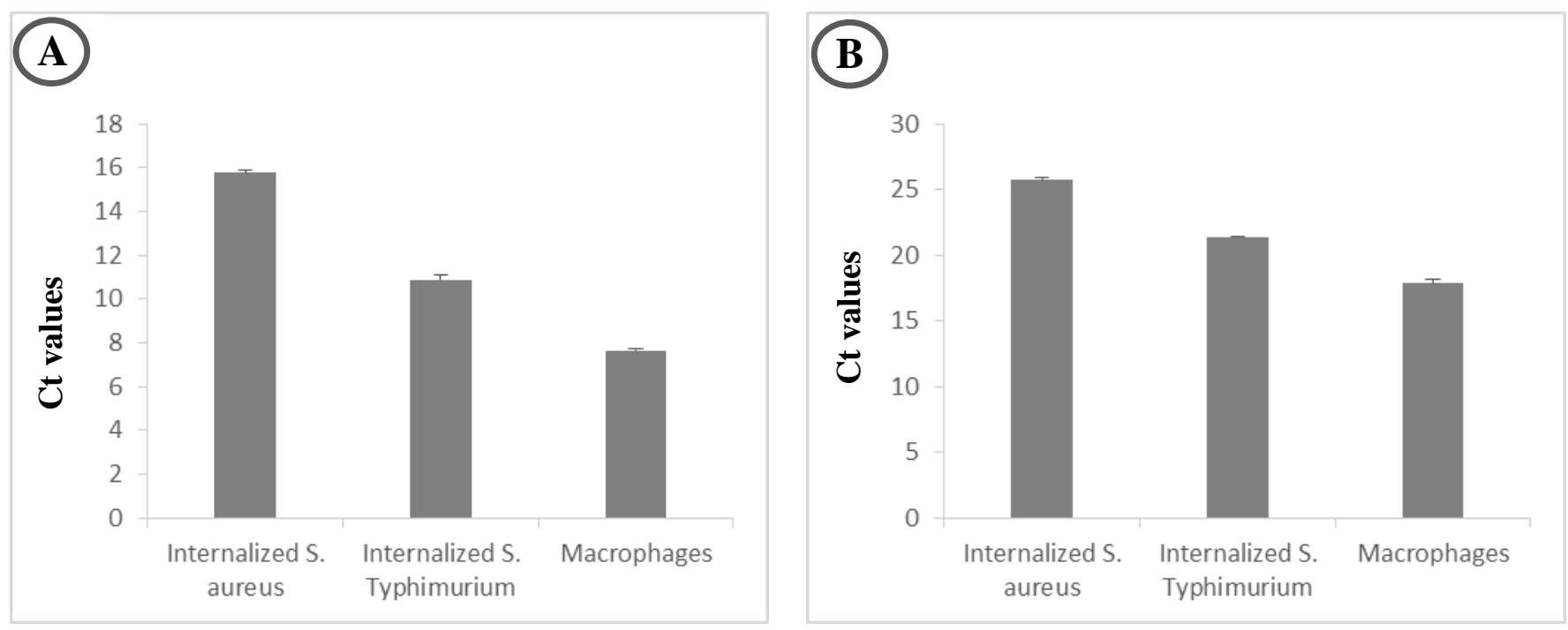

Figure 4: RNA expression levels of selected eukaryotic genes in internalized bacteria samples versus non-infected macrophages, obtained by RT-qPCR. 18S rRNA (A) was picked for its high expression and gapdH mRNA (B) since it is a commonly used eukaryotic referent gene [22].

As described in the introduction, the majority of RNA extraction methods, after host cells uptake of bacteria, use commercial kits for bacterial RNA recovery needed for subsequent analyses. In this report, we describe a simple and inexpensive method to get the same result. A PBS-1\% SDS host cell lysis followed by zirconium beads beating and phenol extraction of RNAs. This method is simple and inexpensive. That method, however, will need optimization when modifying the host cells and bacteria. The method is more efficient on Gram positive bacteria, probably due to different cell walls. Gram negative bacteria cell walls are more fragile than Gram-positive bacteria, with premature lysis of $S$. typhimurium during the extraction leading to premature prokaryotic RNA elimination. A lighter bacterial lysis (using Triton X100), may improve bacterial RNA recovery from $S$. typhimurium. S. aureus RNA extraction is more efficient after their uptake by THP1differenciated macrophages than by Saos-2-osteoblasts. Indeed, RNAs extracted from Saos-2 internalization contain a significant fraction of eukaryotic RNAs and need further optimization. 


\section{Concluding remarks}

The data presented in this report provide a simplified and inexpensive RNA extraction procedure for scientists working on 'host cell-bacteria' interactions that expect to enhance bacterial RNA recovery over the eukaryotic RNA excess from the host cells. This method allows genome-wide expression study of the bacterial RNome during host uptake, without the need to use expensive kits removing the bulk of eukaryotic RNAs. The 'proof of concept' was obtained on a Gram-positive and negative bacterial pathogens used as

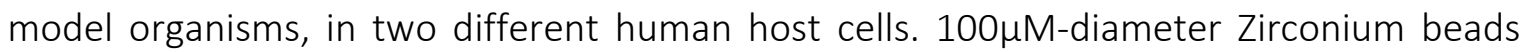
are optimal to get reproducible, clean and intact bacterial RNA extractions from that initial heterogeneous cell mixture. Our protocol is improved from the others because we first use SDS to lyse most of the eukaryotic cells, and then 'high density' Zirconia beads to destruct the bacteria cells embedded within the human cell debris. Therefore, we removed most of the eukaryotic RNAs whereas extracting efficiently the bacterial RNAs from the internalized cells. The amount of eukaryotic mRNAs recovered is low, probably because they are degraded and discarded during the initial lysis step. Eukaryotic rRNAs, however, are detected but their quantity is acceptable for subsequent bacterial gene expression studies.

\section{Funding}

This work was supported by the "Fondation pour la Recherche Médicale" [to S.R., FDM40912]; and Inserm. This work was also funded by the Agence Nationale pour la Recherche (ANR) (grant \# ANR-15-CE12-0003-01 "sRNA-Fit" to B.F.), by the Fondation pour la Recherche Médicale (FRM) (grant \# DBF20160635724 "Bactéries et champignons face aux antibiotiques et antifongiques") to B.F.

\section{References.}

[1] S.S. Chatterjee, H. Hossain, S. Otten, C. Kuenne, K. Kuchmina, S. Machata, E. Domann, T. Chakraborty and T. Hain, Intracellular Gene Expression Profile of Listeria monocytogenes, Infect Immun. 74 (2006) 1323-38.

[2] M.-V. La, D. Raoult and P. Renesto, Regulation of whole bacterial pathogen transcription within infected hosts, FEMS Microbiol Rev. 32 (2007) 440-60.

[3] A.J. Westerman, K.U. Förstner, F. Amman, L. Barquist, Y. Chao, L.N. Schulte, L. Müller, R. Reinhardt, P.F. Stadler and J. Vogel, Dual RNA-seq unveils noncoding RNA functions in hostpathogen interactions, Nature 529 (2016) 496-501.

[4] M.S. Humphrys, T. Creasy, Y. Sun, A.C. Shetty, M.C. Chibucos, E.F. Drabek, C.M. Fraser, U. Farooq, N. Sengamalay, S. Ott, H. Shou, P.M. Bavoil, A. Mahurkar and G.S. Myers, Simultaneous transcriptional profiling of bacteria and their host cells, PLoS One 8 (2013) e80597. 
[5] J. Heptinstall, Isolation of total RNA from bacteria, Methods Mol Biol. 86 (1998) 47-53.

[6] M. Ares, Bacterial RNA Isolation, Cold Spring Harbor Protoc. 2012 (2012) ISSN: 19403402.

[7] W. Ablain, S. Hallier Soulier, D. Causeur, M. Gautier and F. Baron, A simple and rapid method for the disruption of Staphylococcus aureus, optimized for quantitative reverse transcriptase applications: Application for the examination of Camembert cheese, Dairy Sci. Technol. 89 (2009) pp 69-81.

[8] D.C. Rio, M. Ares Jr, J.G. Hannon and T.W. Nilsen, Purification of RNA using TRIzol (TRI reagent), Cold Spring Harb Protoc. doi: 10.1101/pdb.prot5439.

[9] J. Sambrook and D.W. Russell, Purification of Nucleic Acids by extraction with Phenol:Chloroform, Cold Spring Harb Protoc. 2006, doi: 10.1101/pdb.prot4455.

[10] J.M. Voyich, K.R. Braughton, D.E. Sturdevant, A.R. Whitney, B. Saïd-Salim, S.F. Porcella, R. Daniel Long, D.W. Dorward, D.J. Gardner, B.N. Kreiswirth, J.M. Musser and F.R. DeLeo, Insights into Mechanisms Used by Staphylococcus aureus to Avoid Destruction by Human Neutrophils, J . Immunol. 175 (2005) 3907-3919.

[11] C. Garzoni, P. Francois, A. Huyghe, S. Couzinet, C. Tapparel, Y. Charbonnier, R. Renzoni, S. Lucchini, P.D. Lew, P. Vaudaux, W.L. Kelley and J. Schrenzel, A global view of Staphylococcus aureus whole genome expression upon internalization in human epithelial cells, BMC Genomics 8 (2007) 171.

[12] F. Di Cello, Y. Xie, M. Paul-Satyaseela and K.S. Kim, Approaches to Bacterial RNA Isolation and Purification for Microarray Analysis of Escherichia coli K1 with Human Brain Microvascular Endothelial Cells, J Clin Microbiol. 43 (2005) 4197-4199.

[13] G. Gelderman and L.M. Contreras, Discovery of Posttranscriptional Regulatory RNAs Using Next Generation Sequencing Technologies, in: H.S. Alper (ed.), Systems Metabolic Engineering. Methods in Molecular Biology (Methods and Protocols), Totowa, 2013, 985: 269285.

[14] C. Wolz, C. Goerke, R. Landmann, W. Zimmerli and U. Fluckiger, Transcription of Clumping Factor A in Attached and Unattached Staphylococcus aureus In Vitro and during Device-Related Infection, Infect Immun. 70 (2002) 2758-62.

[15] I. Hautefort, A. Thompson, S. Eriksson-Ygberg, M.L. Parker, S. Lucchini, V. Danino, R.J.M. Bongaerts, N. Ahmad, M. Rhen and J.C.D. Hinton, During infection of epithelial cells Salmonella enterica serovar Typhimurium undergoes a time-dependant transcriptional adaptation that results in simultaneous expression of three type 3 secretion systems, Cell Microbiol. 10 (2008) 958-984. 
[16] S. Eriksson, S. Lucchini, A. Thompson, M. Rhen and J.C.D. Hinton, Unravelling the biology of macrophage infection by gene expression profiling of intracellular Salmonella enterica, Mol Microbiol. 47 (2003) 103-18.

[17] H. Gu, C. Zhao, T. Zhang, H. Liang, X.-M. Wang, Y. Pan, X. Chen, Q. Zhao, D. Li, F. Liu, C.-Y. Zhang and K. Zen, Salmonella produce microRNA-like RNA fragment Sal-1 in the infected cells to facilitate intracellular survival, Sci Rep. 7 (2017) 2392.

[18] S.E. Duthier and L.L. Lorenze, Staphylococcal Coagulase: Mode of Action and Antigenicity, J. gen. Microbiol. 6 (1952) 95-107.

[19] M.T. Koskinen, J. Holopainens, S. Pyörälä, P. Bredbacka, A. Pitkälä, H.W. Barkema, R. Bexiga, J. Roberson, L. Sølverød, R. Piccinini, D. Kelton, H. Lehmusto, S. Niskala and L. Salmikivi, Analytical specificity and sensitivity of a real-time polymerase chain reaction assay for identification of bovine mastitis pathogens, J. Dairy Sci. 92 (2009) 952-959

[20] N.H. Smith, P.B. Crichton, D.C. Old and C.F. Higgins, Ribosomal-RNA patterns of Escherichia coli, Salmonella typhimurium and related Enterobacteriaceae, J.Med.Microbiol. 25 (1988) 223-228.

[21] H. Le Pabic, N. Germain-Amiot, V. Bordeau and B. Felden, A bacterial regulatory RNA attenuates virulence, spread and human host cell phagocytosis, NAR 43 (2015) 9232-48.

[22] B. Kozera and M. Rapacz, Reference genes in real-time PCR, J. Appl Genetics 54 (2013) 391-406.

\section{Supplemental Data.}

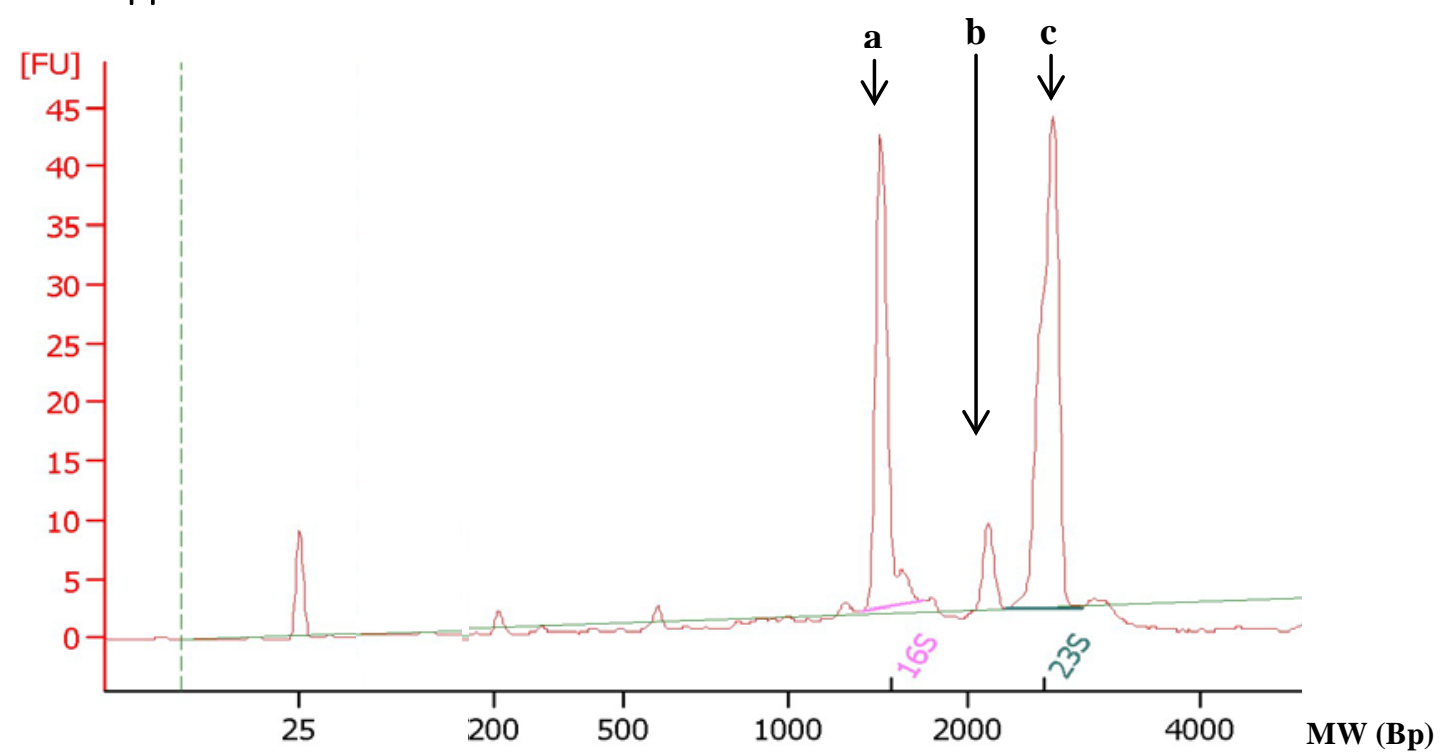

Supplemental Figure A.1: Electrophoregram of the extracted RNAs after $S$. aureus internalized by THP1-macrophage cells. $a=16 \mathrm{~S}$ rRNA,$\quad b=23 \mathrm{~S}$ rRNA degradation, $\quad c=23 \mathrm{~S}$ rRNA. 


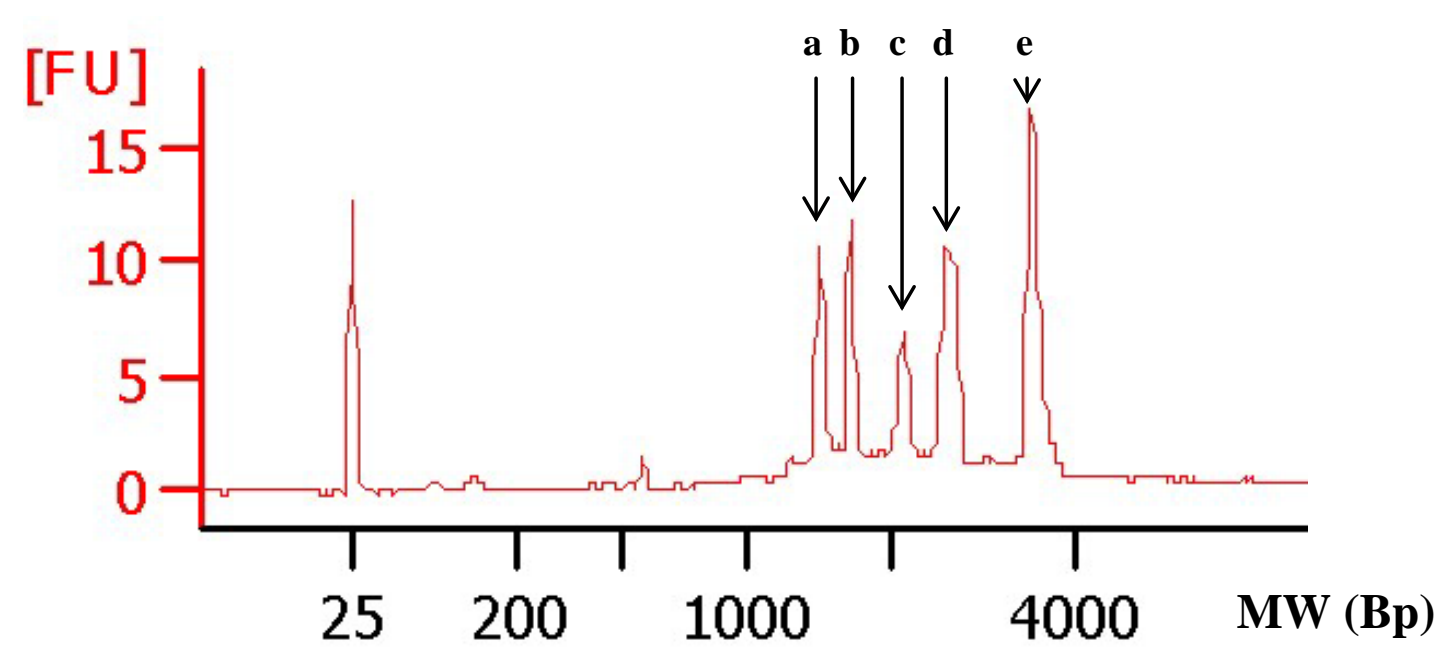

Supplemental Figure A.2: Electrophoregram of the extracted RNAs after $S$. aureus internalization by Saos-2-osteoblast cells.

$a=16 S$ rRNA,$b=18$ S rRNA,$c=23 S$ rRNA degradation, $d=23 S$ rRNA, $e=28$ S rRNA.

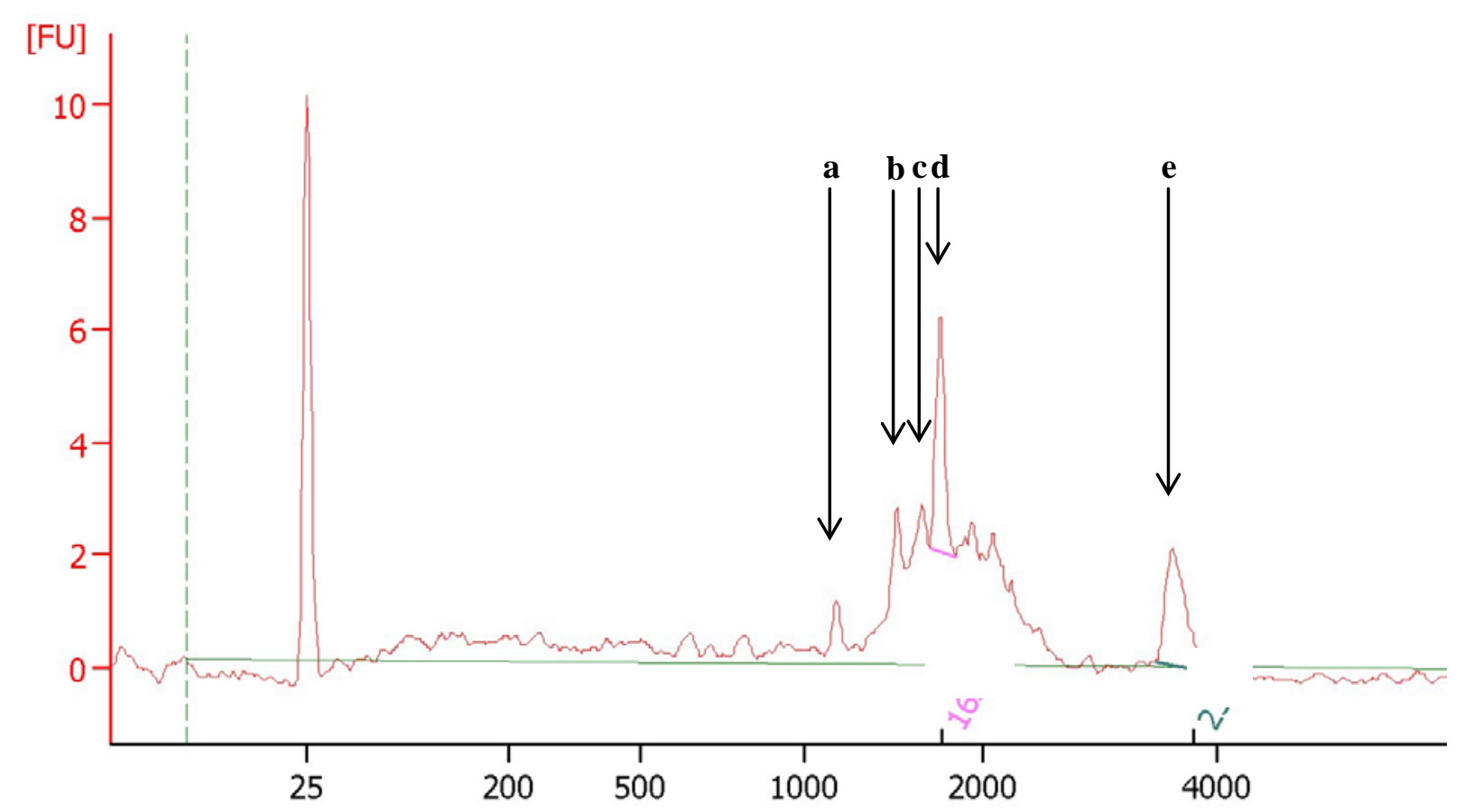

Supplemental Figure A.3: Electrophoregram of the extracted RNAs after S. typhimurium internalization by THP1-macrophage cells.

$a=23 \mathrm{~S}$ rRNA fragment $(1.1 \mathrm{~kb}), \mathrm{b}=16 \mathrm{~S}$ rRNA, $c=23 \mathrm{~S}$ rRNA larger fragment $(1.7 \mathrm{~kb}), \mathrm{d}=18 \mathrm{~S}$ rRNA, e $=28$ S rRNA. 


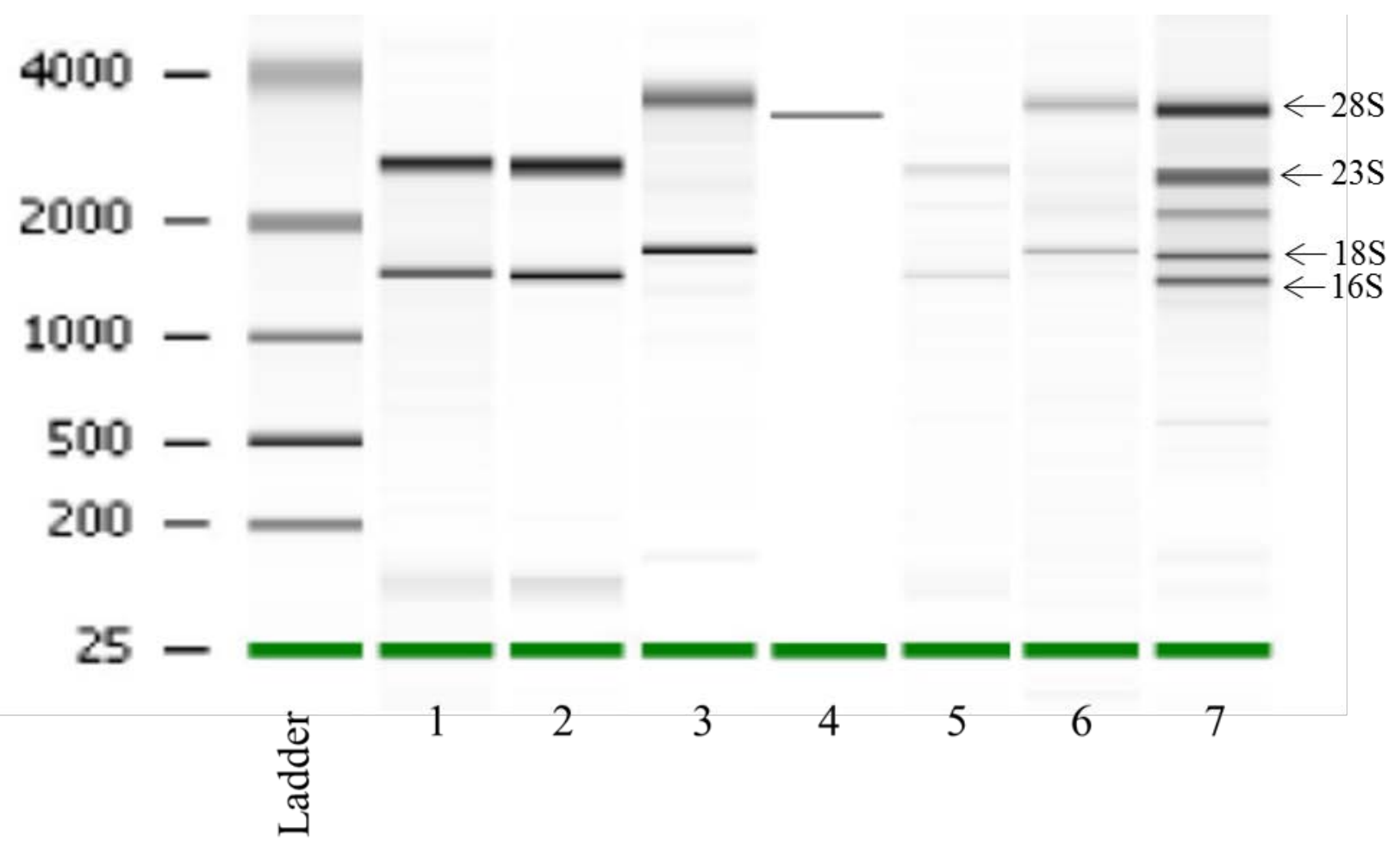

Supplemental Figure B.1: Summary of the RNA chip with S. aureus samples

$1=S$. aureus control extract with glass beads,

$2=S$. aureus control extract with zirconium beads,

$3=$ non-infected macrophages extract with Qiagen kit,

4 = macrophages-internalized $S$. aureus extract with glass beads,

$5=$ macrophages-internalized $S$. aureus extract with zirconium beads,

6 = Saos-2-internalized S. aureus extract with glass beads,

7 = Saos-2-internalized $S$. aureus extract with zirconium beads. 


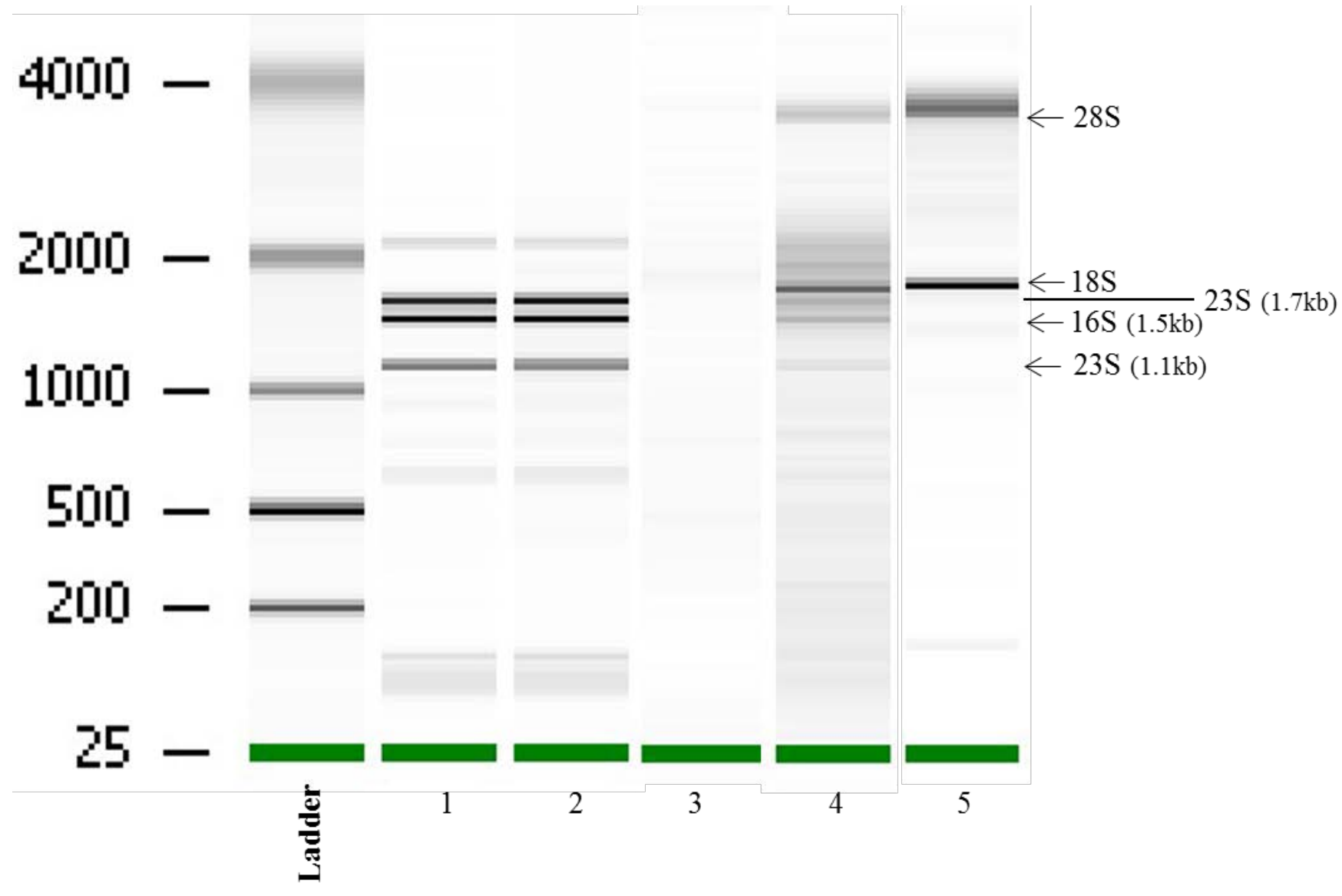

Supplemental Figure B.2: Summary of the RNA chip with S. typhimurium samples.

$1=S$. typhimurium control extract with glass beads,

$2=$ S. typhimurium control extract with zirconium beads,

$3=$ macrophages-internalized S. typhimurium extract with glass beads,

$4=$ macrophages-internalized $S$. typhimurium extract with zirconium beads,

$5=$ non-infected macrophages extract with Qiagen kit. 